\title{
Digital Radio Broadcasting for Community Radios Using Pseudo-random Codes.
}

\author{
Fabrício de Araújo Carvalho, Fernando Walter \\ Departamento de Telecomunicações, Instituto Tecnológico de Aeronáutica, São José dos Campos, Brazil \\ Email: eng.fabricio.carvalho@hotmail.com, fabricio@ita.br, fw2@ita.br
}

Received July, 2013

\begin{abstract}
This paper aims to present a digital radio broadcasting system that explores the advantages of pseudo-random codes. In this context, a transmitter and its dual receiver are able to reuse frequency spectrum bands without interfering on other existing communication systems. It is proposed a communication system that allows radio broadcasting with the following characteristics: lower transmission power, new communication channels and digital signal processing techinques to add positioning services in two dimensions.
\end{abstract}

Keywords: Digital Radio Broadcasting; Software Defined Radio; Spectral Efficiency; Trilateration Positioning

\section{Introduction}

Since 2005, the Brazilian Ministry of Communications has opened a public call to assess the existing digital radio systems. Thus, a possibility arose for the development of a national digital communication system to assist or complement the existing AM/FM modulations already known.

The current AM/FM analog radios are generally considered as a means of entertainment, but are also important in many areas of public life. In this sense, the lower power community radio is an important tool for integration and social development within neighborhoods, as it allows the union of their listeners around their common needs. The awareness raised about current problems motivates social agents to find the most appropriate solutions according to the common interests. Neighborhood radio broadcasting promotes good socio-environmental development and consequently improvement of living standards in the region. Yet, the shortage of available channels has been seen as an impediment for the dispersion of these radios, causing the proliferation of clandestine ones.

The FM receiver has a feature known as capture effect. This occurs when there are more signals transmitted on the same carrier frequency, in this case the FM receiver will respond to the higher power signal and ignore the others.

Frequency bands in communication systems are allocated according to the type of application and the transmission media used. The frequency spectrum used by all these means of communication has become a scarce natural resource. By observing the useful frequency spectrum (Figure 1), we can see a shortage of available bands in comparison to the current demand.

Many countries have already modified their conventional radios or are still using them in parallel with the new digital radio systems. These systems resulted from experiments carried out by universities and research institutions. Some of the best-known digital radio systems have been tested by the Brazilian government (IBOC - In Band On Channel; DRM - Digital Radio Mondiale). Tests with the American and European systems will define the best project in accordance with Brazil's reality. For this reason, it is ultimately important for Brazil to define its own pattern.

Initially, the proposed modulation was exclusively applied to community digital radio broadcasting to provide new communication channels with or without minimal interference as possible on the other ones.

The modulation proposed has the following characteristics: it provides more communication channels in addition to the already existing ones; lower power required

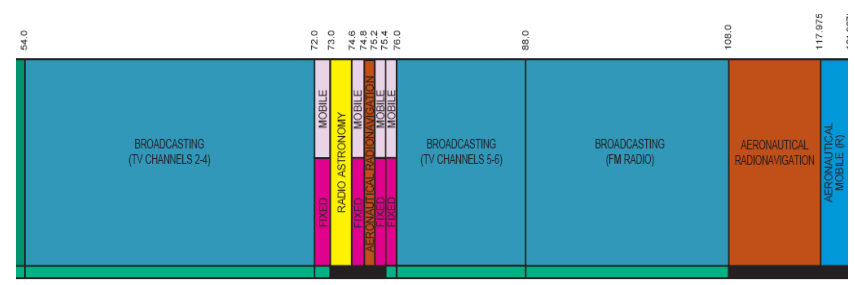

Figure 1. Frequency Allocation: 54.0 - 117.975 MHz (Source: National Telecommunications and Information Administration, http://www.ntia.doc.gov/osmhome/allochrt. html). 
for transmission; high bit rate; positioning service in two dimensions due to the use of pseudo-random codes, PRNs.

\section{Digital Radio}

The PRN codes are used in uncountable communication systems to allow access through multiple channels to the medium using the same carrier frequency. This technique for frequency sharing is known as CDMA.

PRN codes are also used in satellite positioning and navigation to determine position, speed and moving time through a process known as trilateration. Position determination is much easier with these codes because phase ambiguity is reduced by presenting long periods. One of the best-known positioning systems with this method is the GPS system.

Trilateration process of signal is to determine the position of the mobile body. It is based on measuring the propagation time of the Radio Broadcasting station signal TxN up to the receiver. In this case, it is necessary that the stations are synchronized. This synchronization can be achieved when each station is capable of reading a pilot signal of its neighboring stations.

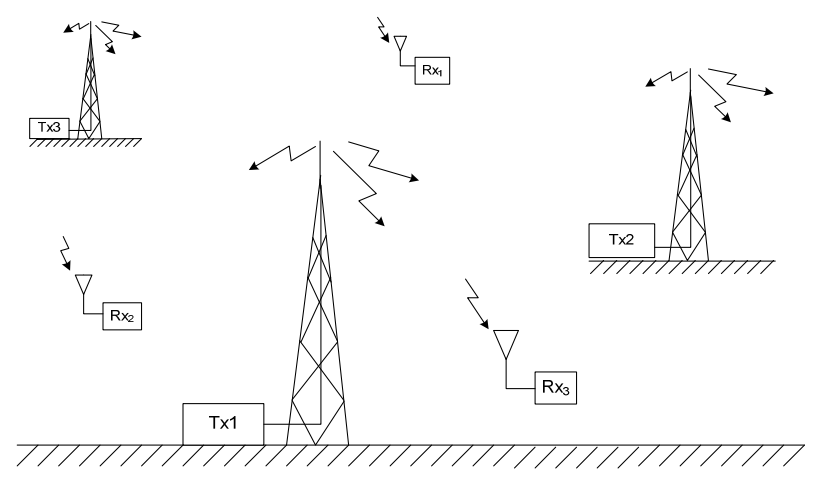

Figure 2. Digital radio broadcasting.

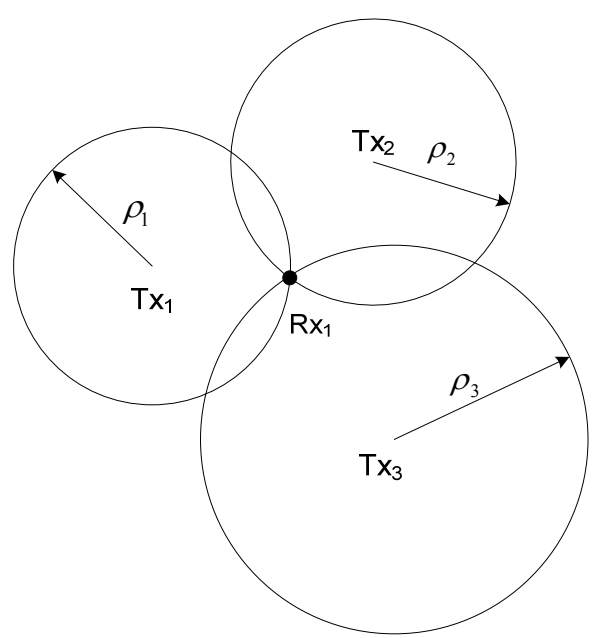

Figure 3. Digital radio broadcasting.

\section{PRN Codes}

The PRN codes used in the radio station are called Gold codes. These are almost orthogonal, thus there is little similarity among them.

They are formed by a sequence of binary "0s" and "1s". In the pseudo-random sequences, the bits are called "chips". The technical term "chip" is used to distinguish the code bit from the information bit. We can see in Equation 1 the PRN code representation $c_{i}(t)$ of the $i-t h$ transmitter.

$$
c_{i}(t)=\sum_{l=0}^{N-1} c_{i l} \prod\left[\frac{t-l T_{\text {chip }}}{T_{\text {chip }}}\right], c_{i l}=0 \text { or } 1
$$

where $c_{i l}$ corresponds to the chip value ("0" and "1") for a given $l$; and $l$ is a counter from 0 to $\mathrm{N}-1 ; \mathrm{N}$ is the number of chips of the code, and $\mathrm{T}_{\text {chip }}$ is the chip duration.

The sequence $c_{i}(t)$ is periodic (Equation 2), of $T_{p}$ pe$\operatorname{riod}\left(\mathrm{N} \times \mathrm{T}_{\text {chip }}\right)$

$$
c_{i}(t)=\sum_{k=0}^{\infty} \sum_{l=0}^{N-1} c_{i l} \prod\left[\frac{t-l T_{\text {chip }}-k T_{p}}{T_{\text {chip }}}\right]
$$

The sequence can be converted into $C_{i}(t)$, of " $1 \mathrm{~s}$ " and "- $1 \mathrm{~s}$ ", if the multiplication operation is used for the signal modulation (Equation 3):

$$
C_{i}(t)=\cos \left[c_{i}(t) \cdot \pi\right]
$$

The sequence is generated using two maximum length registers called G1 and G2 (Figure 4). For a sequence with a length of 63 chips $\left(=2^{6}-1\right)$, registrers with 6 cells or elements are used. Both records are initialized within 1s (module 2 operation). Each record has its values shifted according to a time reference $(10.23 \mathrm{MHz})$, which will determine the chip rate and hence the code period.

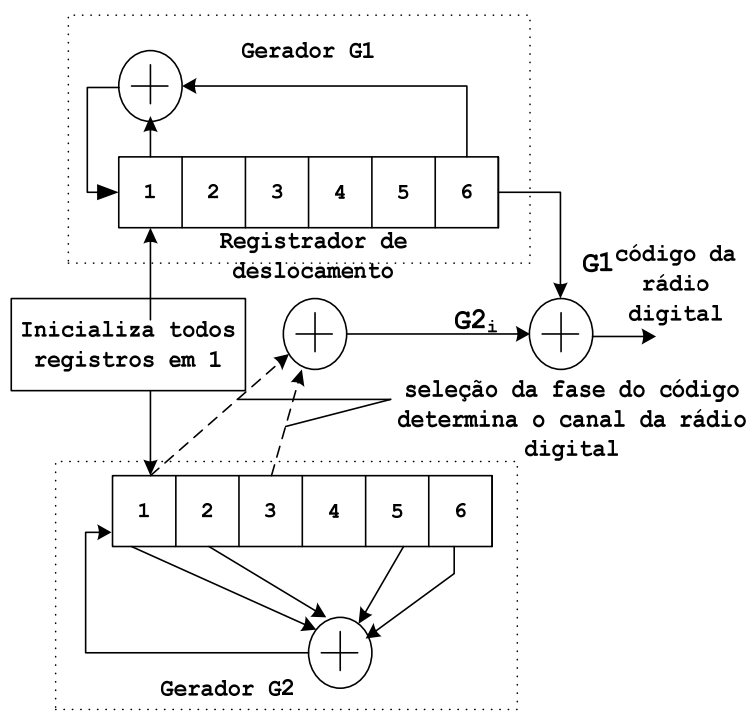

Figure 4. Gold code generator. 
Table 1 shows polynomial G1(t) and G2(t) used to match each other, the value in each element of the registers G1 and G2 through a module 2 operation $(\oplus)$.

The PRN sequence determines which station will be received. The distinction made by the receiver between the information from the transmitters is made through a correlation process. The correlation occurs between the code contained in the signal transmitted by the broadcasting station and its reply, present in the target receptor.

Correlation makes possible to measure the degree of similarity between these signals by amplitude of correlation. The amplitude of correlation between distinct and orthogonal PRNs is approximately equal to zero, $\mathrm{R}_{\mathrm{ij}}(\tau)$ (cross-correlation) for every $\tau$ delay (Equation 4 ).

$$
\begin{aligned}
& R_{i j}(\tau)= \\
& \frac{1}{N T} \int_{0}^{N T_{\text {chip }}} C_{i}(t) C_{j}(t+\tau) d t \cong 0 ; p / \text { qualquer }|\tau|
\end{aligned}
$$

In this equation, $\mathrm{C}_{\mathrm{i}}(\mathrm{t})$ and $\mathrm{C}_{\mathrm{j}}(\mathrm{t})$ are the PRN codes for the $\mathrm{i}$-th and $\mathrm{j}$-th transmitter, respectively. For the autocorrelation (same codes), $\mathrm{R}_{\mathrm{ii}}(\tau)$, the amplitude is different from zero for delay values $\tau$ below one chip; $0 \leq|\tau| \leq$ $\mathrm{T}_{\text {chip }}$ (Figure 5):

As we can observe, the autocorrelation peaks have a width of two chips and repeat every $\mathrm{T}_{\mathrm{C}}$ period of the code $\left(=\mathrm{N} \mathrm{x} \mathrm{T}_{\text {chip }}\right)$. The amplitude increases linearly from a previous chip to a maximum in the alignment, decreasing to zero, one chip after the maximum. For orthogonal codes, the amplitude $\mathrm{R}_{\mathrm{ii}}(\tau)$ will be approximately zero for $\tau$ delays larger than the chip (Equation 5).

$$
\begin{aligned}
R_{i i}(\tau) & =\frac{1}{N T_{\text {chip }}} \int_{0}^{N T_{\text {chip }}} C_{i}(t) C_{i}(t+\tau) d t \\
& = \begin{cases}A^{2}\left(1-\frac{|\tau|}{T_{c}}\right) ; & \text { para }|\tau|<T_{\text {chip }} \\
\cong 0 ; & \text { para }|\tau|>T_{\text {chip }}\end{cases}
\end{aligned}
$$

Figure 6 shows the chip sequence provided by the PRN code generator (Figure 4).

The autocorrelation for this code is shown in Figure 7. We can observe the maximum amplitude-normalized values $[63,15,-1,-17]$. The sequence has a 21 chips delay only to emphasize the maximum amplitude.

Figure 8 shows the frequency spectrum of the 10.23 $\mathrm{MHz}$ PRN sequence sampled at a rate of $60 \mathrm{MHz}$.

Tabel 1. PRN code generator polynomials.

\begin{tabular}{cc}
\hline Reg. & Polinomyal \\
\hline G1 $(\mathrm{t})$ & $1 \oplus x^{1} \oplus x^{6}$ \\
$\mathrm{G} 2(\mathrm{t})$ & $1 \oplus x^{1} \oplus x^{2} \oplus x^{5} \oplus x^{6}$ \\
\hline
\end{tabular}

Both transmitter and receiver are designed and encoded with the Matlab ${ }^{\circledR}$ software for the concept tests. These signals were generated in baseband and intermediate frequency (IF) with signal processing.

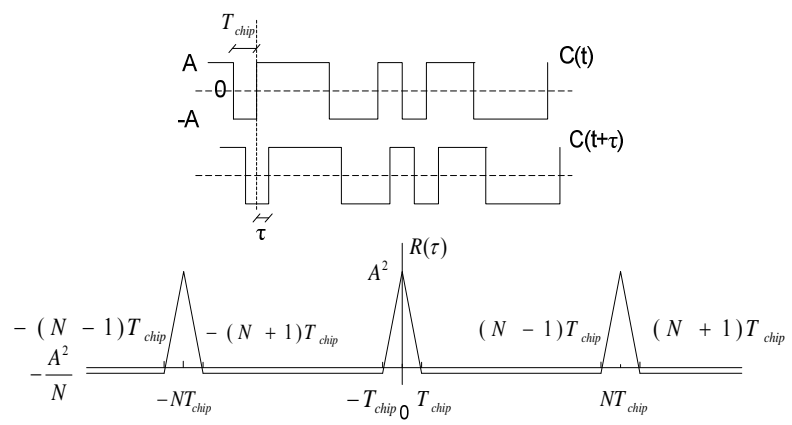

Figure 5. PRN sequence autocorrelation process.

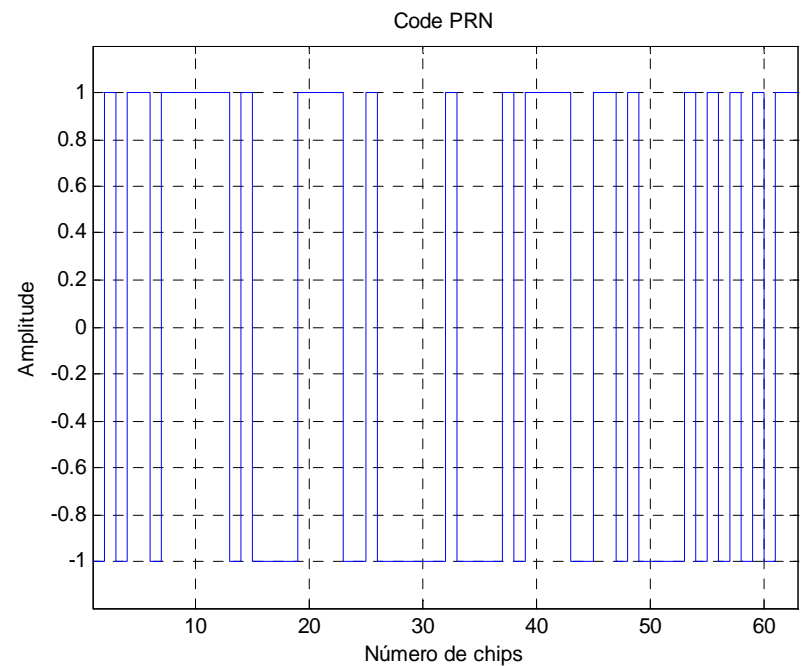

Figure 6. PRN sequence of 63 chips from the Gold code generator.

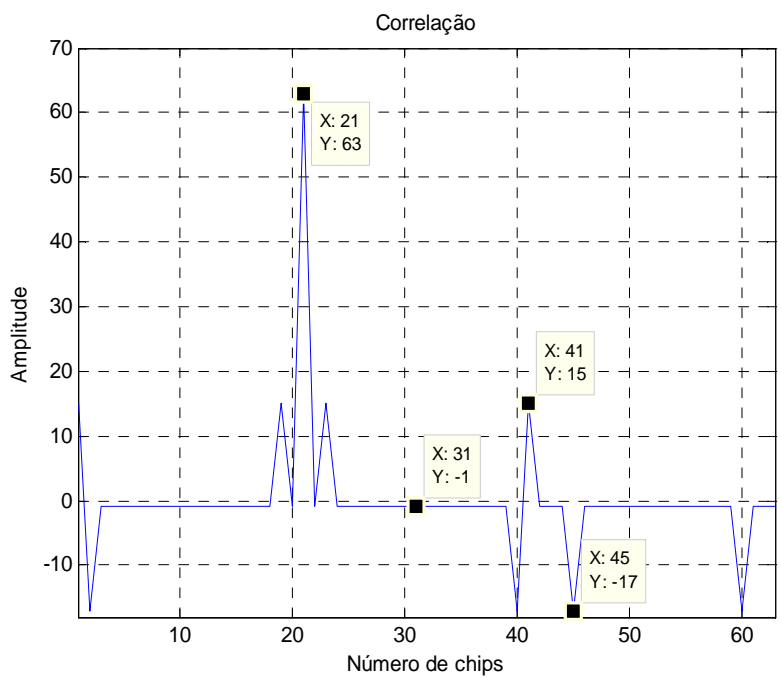

Figure 7. PRN code autocorrelation with 21 chips delay. 


\section{CDMA Transmitter}

Before The block diagram of Figure 9 provides an overview of the transmitter encoded in baseband and digital IF. In this diagram, information (voice signal) is digitized by an A/D converter. The binary sequence provided by the converter modulates the PRN sequence (in +1 and -1 ). This one will convey information. Subsequently, this signal is heterodyned for an IF after being multiplied by a digital carrier. If necessary, the transmission rate can be increased by adding another component in quadrature.

O sinal transmitido é então armazenado em um arquivo binário para ser lido pelo receptor em software.

The signal transmitted is thereafter stored in a binary file to be read by the software receiver.

\section{CDMA Receirver}

After Figure 10 shows a common CDMA receiver with a block diagram.

After downconverter, the analog IF is sampled, quantized and encoded by an A/D converter. Figure 11 illustrates the sampling in the $f_{\mathrm{s}}$ frequency $(60 \mathrm{MHz})$.

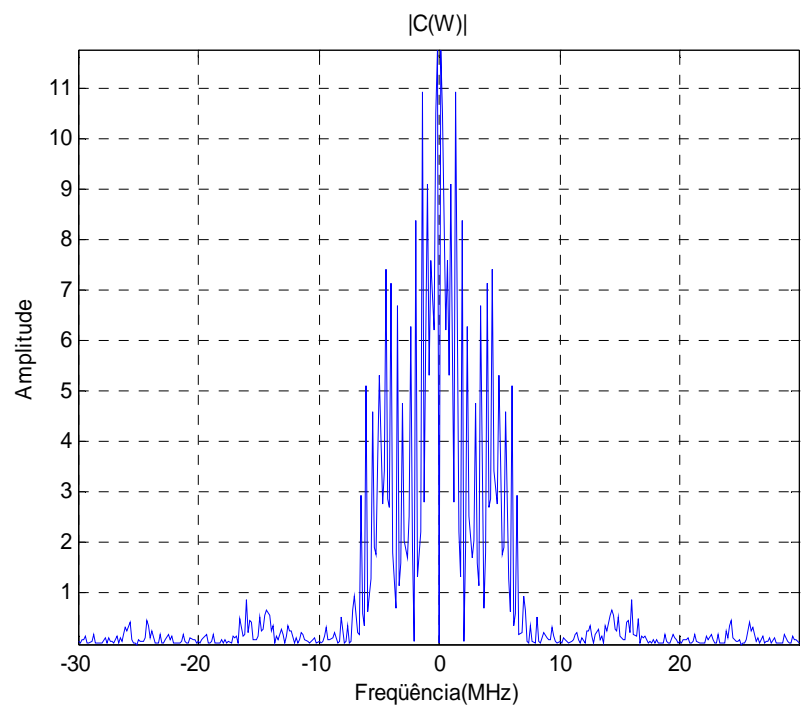

Figure 8. Frequency spectrum for the PRN code, already sampled.

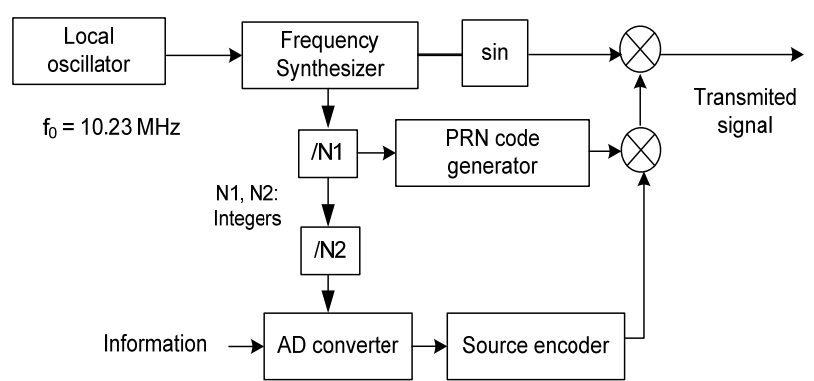

Figure 9. Transmitter block diagram in baseband and digital IF.
The converter's output is a digital IF that will be processed by the correlator using programming techniques. Figure 12 shows the tracking process executed by the CDMA receiver [1-4] through a block diagram.

\section{Results}

The The audio signal captured by the computer's microphone was sampled at a rate of $8 \mathrm{kHz}$, quantized and encoded into 16-bit words. Each information bit modulates the phase of a PN code to $\pm \pi$ rad. This one modulates the $10.7 \mathrm{MHz}$ intermediate frequency and stores it in the accumulator ("buffer"). The signal transmitted is a BPSK (Binary Phase Shift Keying). For bit timing, it is

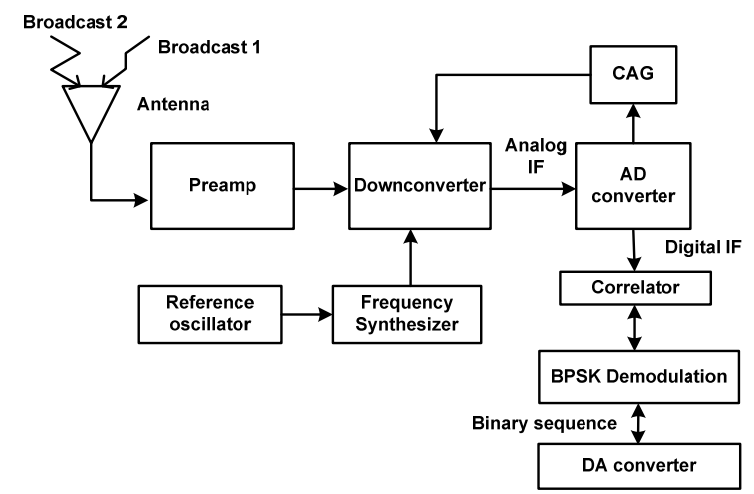

Figure 10. Community radio broadcasting CDMA receiver.

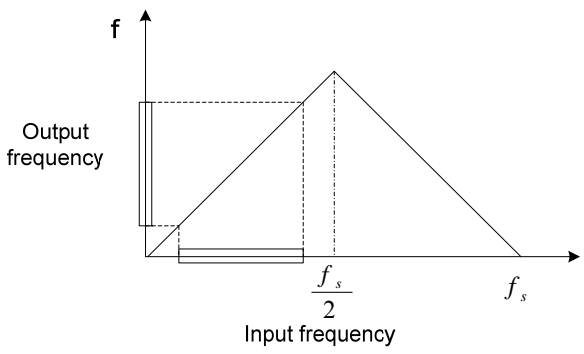

Figure 11. Bandpass sampling process.

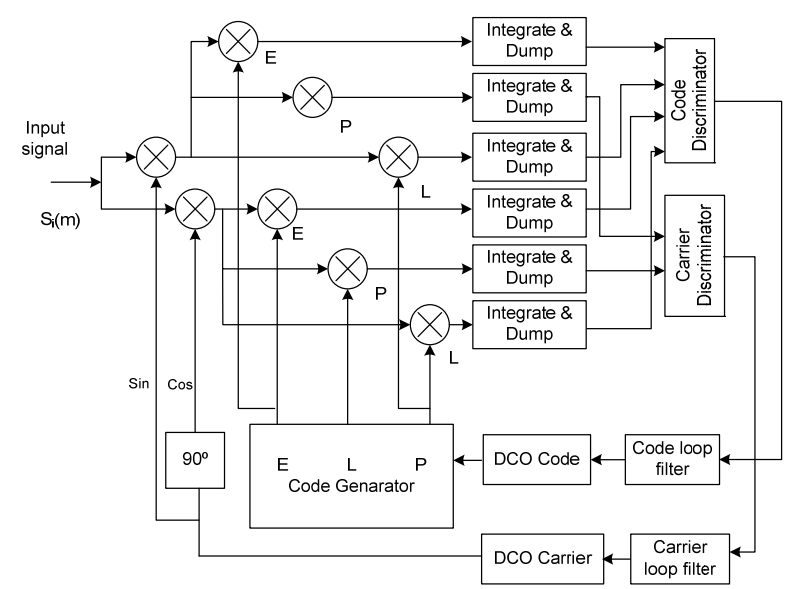

Figure 12. Tracking and demodulation step. 
transmitted the following preamble 10101010101010101 for every five samples of the audio signal.

For the transmitter screen, we have the following windows: 1: audio signal (counting from 1 to 5); 2: audio signal spectrum; 3: signal spectrum in the digital IF; 4 : truncated PRN code ; 5: Doppler on the carrier; 6: digitised IF; 7: error in carrier phase; 8: 16-bit word (quantified and encoded audio), 9: error in frequency code; 10: BPSK signal transmitted and 11: error in code phase.

Figure 14 shows the signal stored in binary file and processed by the receiver.

The station's signal must be weak enough to not affect other communication systems. In this project, it is transmitted with power next to the thermal noise.

Therefore, the broadcasting station reduces its interference on other communication systems.

On the receiver's screen we can list the following windows 1: Doppler on the carrier; 2: error in carrier phase; 3: diagram of the constellation; 4: error in the code frequency; 5: phase error of the code; 6: recovered bit sequence; 7: correlation amplitude of the advanced signal; 8: correlation amplitude of the aligned signal, and 9: correlation amplitude of the delayed signal.
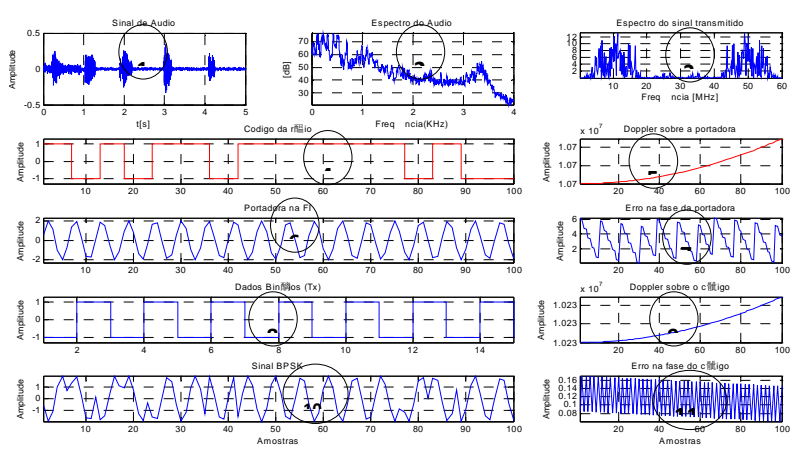

Figure 13. Community radio broadcasting CDMA transmitter. Monitoring interface.
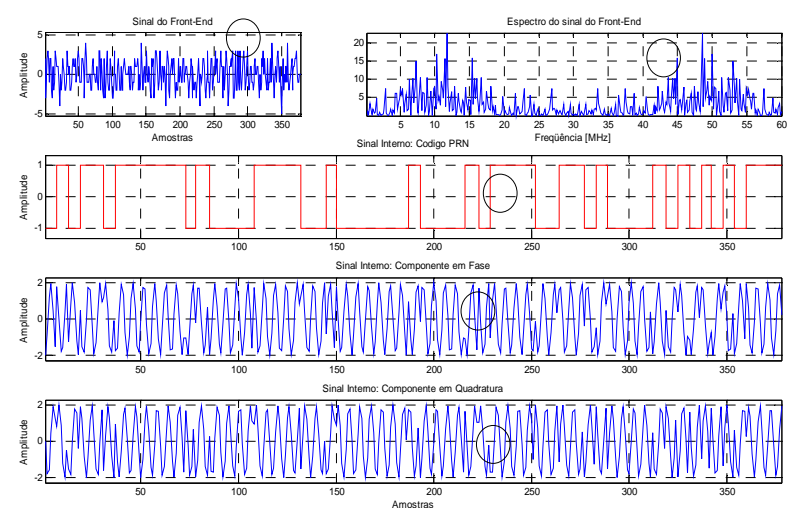

Figure 14. Tracking step signals: 1: received signal; 2: spectrum of the received signal; 3 : internally generated PRN code; 4: PRN code and carrier in phase; 5: PRN code and carrier in quadrature.

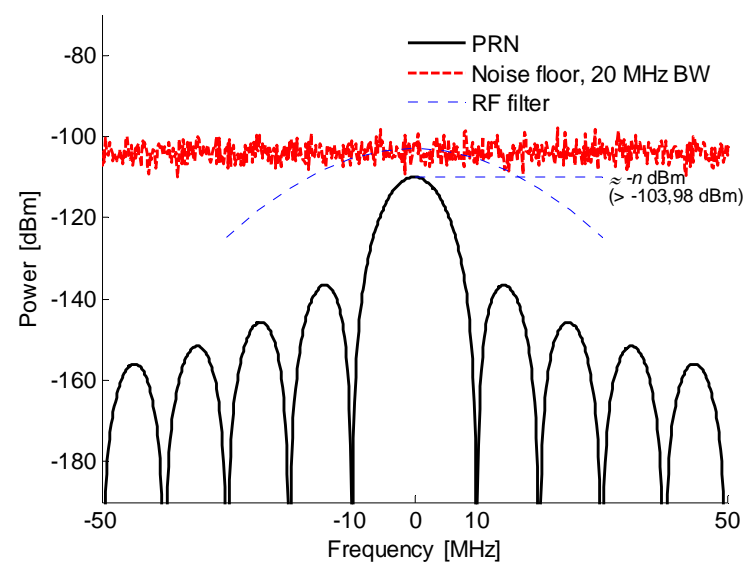

Figure 15. Thermal noise and power spectral density for the received signal.
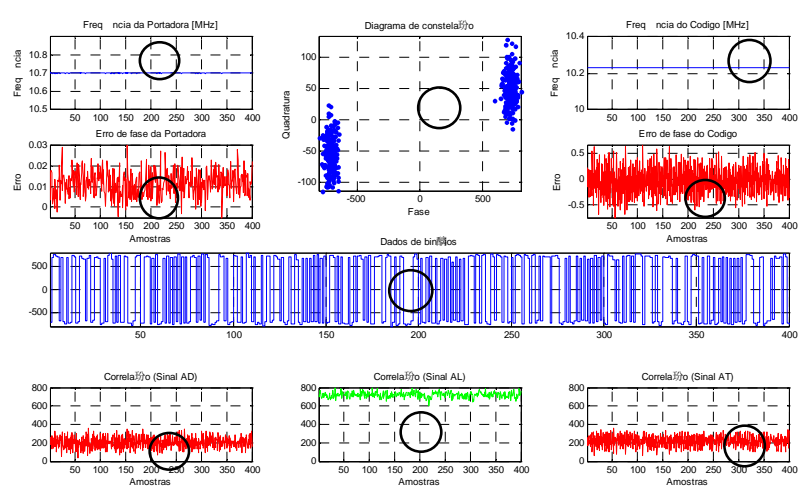

Figure 16. Monitoring interface. Tracking and demodulation. CDMA Community radio broadcasting receiver.

\section{Conclusions}

Headings, Here is proposed a digital radio broadcasting system capable of operating in the same frequency bands allocated for AM /FM radio, but with less transmission power. It proposes to transmit information with power below or closer to the thermal noise, and its value will depend on the bit/second rate chosen for the system, on the code length and on the range desired.

Many current communication systems use high power transmitters to ensure service quality. By using PRN codes, it is possible to transmit signals with low power without interfering on other existing systems (similarly to what happens with GPS).

The use of PRN codes expands the use of the receiver, allowing it to determine its position, once it tracks down the signals from at least three radio stations. In this case, the transmitters must be with synchronized time bases. The positioning service can assist current satellite navigation systems, bringing real benefits for agriculture, administration and public services. In the aviation sector, it is able to assist aircraft landings (e.g. category III) and also serve as a DGPS correction channel DGPS [5] for 
GNSS systems.

From the concept of community radio, this prototype of the source code can be easily adapted to local needs and real time processing by high performance digital signal processors such as DSP and FPGAs and it can be made available for commercial use and for police forces. Because of its low power consumption and alternative channels, it can be used by the Fire Department, Civil Defense, Police, and also the Armed Forces for communication via walk-talk in public safety services. For the Armed Forces, the signal can be easily encrypted and changed periodically.

It must be highlighted that this project would enhance the national production of transmitters/receivers.

\section{Acknowledgements}

The Company NavCon Navegação e Controle by allowing the training of its engineers and by being a partner company of the Instituto Tecnológico de Aeronáutica ITA.

\section{REFERENCES}

[1] J. B. Y. Tsui, Fundamentals of Global Positioning System Receivers, A software approach. Wiley Interscience Publicantion, 2000. doi:10.1002/0471200549

[2] F. A. Carvalho, Alexandre, B. V. Oliveira and E. F. Walter, "Receptor GPS em Software. In: $25^{\circ}$ Simpósio Brasileiro de Telecomunicações - XXV SBrT 2007, Recife, PE, Brasil, set, 03 a 07.

[3] Carvalho and F. A. e F. Walter, "Receptor GPS por Software em Tempo Real. Parte I: Geração dos Sinais", Anais do $12 \mathrm{O}$ Encontro de Iniciação Científica e Pós-Graduação do ITA - XII ENCITA / 2006, São José dos Campos, SP, Brasil, out., 16 a 19.

[4] Carvalho and F. A. e F. Walter, "Receptor GPS Por Software em Tempo Real. Parte II: Correlator", Anais do $12 \mathrm{O}$ Encontro de Iniciação Científica e Pós-Graduação do ITA - XII ENCITA / 2006, São José dos Campos, SP, Brasil, out., 16 a 19.

[5] Parkinson, W. Bradford, Jr. Spilker and J. James, Global Positioning System: Theory and Applications- vol 1. Published by the American Institute of Aeronautics and Astronautics, Inc. Hopfield model. 